\title{
Understanding convolutional neural networks via discriminant feature analysis
}

\author{
HAO XU, YUERU CHEN, RUIYUAN LIN AND C.-C. JAY KUO
}

\begin{abstract}
Trained features of a convolution neural network (CNN) at different convolution layers is analyzed using two quantitative metrics in this work. We first show mathematically that the Gaussian confusion measure (GCM) can be used to identify the discriminative ability of an individual feature. Next, we generalize this idea, introduce another measure called the cluster purity measure (CPM), and use it to analyze the discriminative ability of multiple features jointly. The discriminative ability of trained CNN features is validated by experimental results. Research on CNNs utilizing GCM and CPM tools offers important insights into its operational mechanism, including the behavior of trained CNN features and good detection performance of some object classes that were considered difficult in the past. Finally, the trained feature representation is compared between different CNN structures to explain the superiority of deeper networks.
\end{abstract}

Keywords: Convolutional neural networks, Object recognition, Empirical analysis

Received 23 September 2018; Revised 9 November 2018

\section{INTRODUCTION}

The convolution neural network (CNN) offers excellent object classification performance due to its superior feature representation capability. This is automatically obtained by the backpropagation training process with a large amount of training data. Great efforts have been exerted to analyze the trained filters in order to explain CNN's performance. Existing visualization methods [1-3] reconstruct input images or visualize the responses to provide insights into the mechanism behind the activation of certain filters. Despite partial success in explaining CNN's performance, such analysis is less scalable given an increasing number of object classes and deeper network structures. In [4], the average precision (AP) of an individual filter is computed and used as a quantitative measure of a trained $\mathrm{CNN}$ filter's discriminative ability. An important contribution of their work is to confirm the existence of 'GMC-like features'.

Human brain cells that only respond to specific and complex visual stimuli (such as the face of one's grandmother) are called the grandmother cells (GMC) [5]. They have been studied in artificial neural networks and are believed to play a critical role in object recognition. For example, the cat filter was extensively studied in [6] for

Ming Hsieh Department of Electrical Engineering, University of Southern California, 3740 McClintock Avenue, Los Angeles, CA, USA

Corresponding author:

$\mathrm{Hao} \mathrm{Xu}$

Email: iamxuhao@gmail.com its resemblance to the cat-face GMC. The term 'GMClike features' is used to refer to features or feature groups that respond solely to one object class. A similar concept is adopted in the standard feature encoding for image classification $[7,8]$.

In Fig. 1, two conv_5 filters from the CaffeNet are used to demonstrate the importance of studying the GMC-like features. From left to right, the filters correspond to the dining table' and 'dog' object class, respectively. In the left, no dining table image is among the top nine activations and there is a significant overlap in the Gaussian confusion plot between the filter responses of the dining table and nondining table classes. In the right, all top nine activations are dog images and there is little overlap in the Gaussian confusion plot between the filter responses of the dog and non-dog classes. The visual inspection of the top nine activations in Fig. 1 demonstrates the superior discriminative ability of GMC-like features, but such inspection cannot be conducted in large-scale networks due to the infeasible workload on the human inspector. Note that the Gaussian confusion plot reflects the important message of the top nine activations and, therefore, motivates us to develop the Gaussian confusion measure (GCM, see Section A), which yields a score representing the discriminative ability of a feature without human intervention.

For certain object classes, one CNN feature is not a GMC-like feature by itself (see Section B). However, when a group of features work together, they behave as a GMC-like feature group. To understand the observation, we develop another measure called the cluster purity measure (CPM, see Section B) to gauge the group performance of features. 

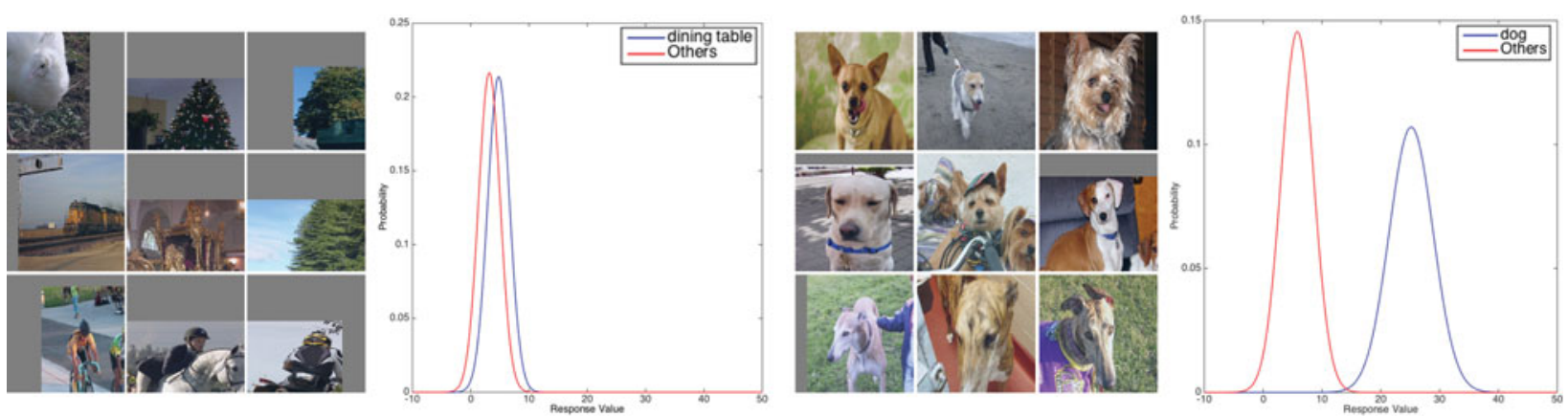

Fig. 1. None-GMC-like and GMC-like features are compared in the left and right subfigures. The first and third subfigures give the top nine activations for two $\operatorname{conv}_{5}$ filters and the second and fourth subfigures show their Gaussian confusion plot, which reflects the distribution of a filter's response value (see discussion in Section A). In this example, the GMC filter (i.e., filter 142 in conv ${ }_{5}$ of the fast-RCNN [9] CaffeNet model) is mainly activated by the dog class and its Gaussian confusion plot shows better separation.

As opposed to training an SVM for a subset of features and evaluating the resulting AP, CPM can be directly computed and not subject to the stochastic difference.

Our research goal is to gain a better understanding of the CNN by evaluating its trained features and studying the GMC-like features. It has three major contributions. First, we propose the GCM to automatically evaluate the performance of an individual filter. Second, we propose the CPM to automatically evaluate the group performance of a set of filters. They are both simple and efficient to train, and are useful in the automatic evaluation of the $\mathrm{CNN}$ features. Third, we study the CaffeNet and the VGG_M_1024 architectures by evaluating its filters using the proposed metrics. Our study provides an explanation of their stellar performance, reveals CNN's capability in detecting certain object (e.g. the dining table, see Section B), identifies potential issues with feature representations, and verifies the benefits of using deeper networks.

The rest of this paper is organized as follows. Related work is reviewed in Section II. Two metrics are introduced in Section III to evaluate whether a filter or a group of filters qualifies as the GMC-like feature. Experimental results based on the evaluation of the GMC-like features are reported in Section IV. Concluding remarks are provided in Section V.

\section{RELATED WORK}

\section{A) GMC-like features}

To better understand CNN trained features and their representations, several methods are proposed to find locally optimal visual inputs for individual filters in $[3,10,11]$. Despite starting from different input priors, these methods were capable of selecting images that maximally activate a filter. By examining visualization results, one can verify the existence of GMC-like features as well as identify the targeted image content associated with the GMC-like feature. Besides visually inspecting features, Agrawal, et al. [4] evaluated $\operatorname{conv}_{5}$ features with respect to their AP on the PASCAL-VOC 2012 dataset and showed the existence of GMC-like features for several object classes.

\section{B) CNN interpretability}

The exact purpose of filters in a convolution layer has been studied since early days of CNN research. It was observed in $[12,13]$ that the conv $_{1}$ features are low-level features such as edge, color, or texture. The deeper convolution layers extract higher-level features by assembling lower level features in the previous layer with different weights [14]. The pooling layers provide spatial and possibly rotation invariance to feature extraction [15]. The features assembly was studied in [16]. It was concluded that only a local subnetwork is activated or trained for a specific input pattern. The selection is done through the local competition of several activation functions such as rectified linear, maxout and local winner-take-all. Research in [17] reveals the CNN model structure by formulating the CNN as a deformable part model, where the convolution layers serve as part-feature detectors and the pooling layer uses a distance transform to assign different weight to partdetectors according to their relative locations. An interesting study conducted in [18] indicates that object detectors could be trained to classify scene images, which have certain similarity as part-detectors are trained to recognize objects.

Kuo [19] and [20] studied the role of the nonlinear activation functions in CNNs. It was argued that the nonlinear activation units act as a rectifier and prevent the sign confusion problem. Without intermediate nonlinear activation units, a negative response at the first layer multiplied by a negative filter weight at the second layer produces the same sign as a positive response multiplied by a positive filter weight. The same confusion occurs between a negative response followed by a positive filter weight, and a positive response followed by a negative filter weight.

Additional insights can be obtained by investigating the vulnerability of CNNs. Adversarial examples [21] are generated by adding imperceptible perturbations to the original test image so that the neural network will make a different prediction. Goodfellow et al. [22] proposed a fast gradient sign method to efficiently compute perturbations. Xie et al. [23] proposed a Dense Adversary Generation algorithm to generate adversarial examples for the semantic segmentation and object detection problems. 
Zhang et al. [24] proposed a technique to detect representation bias of CNNs. Due to dataset bias, unrelated co-appearing features may be used by $\mathrm{CNNs}$ to estimate the target attribute. To detect such representation flaws, the method requires additional manual annotations on the attribute relationship. The attribute relationships encoded by CNNs are extracted and then compared with manually annotated relationships, where disagreements suggest flaws in representations.

Researchers also used interpretable graphs to encode knowledge from pretrained CNN. Zhang et al. [25] built a graph to find the knowledge hierarchy encoded by CNN conv-layers, where each node corresponds to a part. Zhang et al. [26] used the decision tree to obtain interpretable prediction rules of a trained network.

Some recent works aim to build interpretable alternatives to CNNs. Sabour et al. [27] introduced the CapsNet using units named 'capsules' [28]. They are groups of neurons. A capsule is implicitly associated with a visual entity. Instead of a scalar output, each capsule outputs a vector, whose orientation corresponds to certain properties (e.g., size and deformation) of the entity. The length of the vector encodes the probability of existence. The routingby-agreement mechanism allows the model to build a hierarchical relationship between entities. Experiments on the MNIST [29] dataset showed that each dimension of the capsule output vector possessed semantic meanings such as width and stroke thickness. Chen et al. [30] proposed a variant of the generative adversarial network (GAN) named the InfoGAN, which aims to obtain an interpretable representation in an unsupervised manner. It is achieved by maximizing the mutual information between a subset of input noise variables and the generated images. Experiments on the MNIST dataset [29], SVHN dataset [31], 3D image datasets of faces [32] and chairs [33] and CelebA face datasets showed that the subset of latent variables correspond to visual properties such as the digit width in the MNIST dataset, the lighting condition in the SVHN dataset, rotation in the $3 \mathrm{D}$ images, and the hair style in the celebA dataset.

\section{C) Object detection networks}

Since Girshick et al. [12] introduced the RCNN to solve the object detection problem, many efforts have been done to improve its efficiency and accuracy. The spatial pyramid pooling (SPP) was proposed in [34] to share convolution computations. Girshick [9] added the bounding box regression and extended the finetuning to all layers for better accuracy. The time-consuming region proposal extraction was replaced by the region proposal network to speed up detection to near real-time in [35]. Deeper network configurations $[36,37]$ offered even better performance in the benchmarking datasets at the price of a higher training cost with more parameters. Although the performance can be further improved with larger networks, we are more interested in understanding the reason for their success. By understanding the strength and weakness of a network, it helps stretch the performance furthermore at a reasonable cost of added parameters.

\section{EVALUATION METRICS}

To train a CNN for object detection, each training sample contains an object in a region of interest (ROI) with class label $c$. The filter responses in convolution layers indicate trained features. In conv ${ }_{1}$, filters act as low-level feature extractors. As more information is aggregated in deeper convolution layers, filters become more discriminative and class-specific. As a result, filters will be activated by more specific inputs.

The problem of interest can be stated as follows. Given an ROI which has an object inside, we extract one value from the filter response at the conv $v_{5}$ layer by max pooling. There are 256 filters at the conv 5 layer and, consequently, we obtain a response vector of dimension 256 for each input ROI. Our question is whether there exists one or a group of filters that can help separate an object class from other classes. In the following, we propose two metrics that offer a quantitative answer to this question.

\section{A) Gaussian confusion measure}

We use $N$ testing data samples to test a CNN for a specific object class, say, class $c$. The testing samples consist of both positive ones that are in class $c$ and negative ones that are not in class $c$. To analyze a specific filter, we take its response value from the $256-\mathrm{D}$ response vector and collect $N$ response values, each is associated with a class label. They can be classified into two groups according to their class labels; namely, in class $c$ or not in class $c$. Consequently, we can draw two distribution curves of response values for the two groups. This explains the Gaussian confusion plots in Fig. 1, where the $y$-axis is the normalized histogram value and the $x$-axis is a specific filter response value. As shown in Fig. 1, each filter response can be well approximated by a Gaussian (or normal) function denoted by $\mathbb{N}(m, \sigma)$, where $m$ is the mean and $\sigma$ is the standard deviation.

We can state the problem mathematically below. For a set of testing samples $\left(\vec{x}_{i}, y_{i}\right)$, where $\vec{x}_{i}$ is the ROI and $y_{i}$ is the class label, we use $\mathbb{F}_{i}\left(f_{k j}\right)$ to denote the response value of $f_{k j}$ which is the $j$ th filter in the $k$ th convolution layer. We group the response values according to their class labels into $\mathbb{C}$ groups, where $\mathbb{C}$ is the number of class labels in the testing dataset.

To get the GCM from a given filter, $f_{k j}$, and a given object class, $c \in \mathbb{C}$, we need to compute the mean and standard deviation of two hypotheses: $H_{\mathrm{o}}$ and $H_{1}$, where $H_{\mathrm{o}}$ states that the object belongs to class $c$ while $H_{1}$ states that the object does not belong to class $c . N_{\mathrm{o}}$ and $N_{1}$ indicate the number of objects belonging to the two hypotheses, respectively.

The mean and standard deviation of hypothesis $H_{\mathrm{o}}$ can be computed as

$$
m_{\mathrm{o}}\left(f_{k j}, c\right)=\frac{1}{N_{\mathrm{o}}} \sum_{\forall i, \text { s.t. } y_{i}=c} \mathbb{F}_{i}\left(f_{k j}\right),
$$




$$
\sigma_{o}\left(f_{k j}, c\right)=\sqrt{\frac{1}{N_{\mathrm{o}}} \sum_{\forall i, s . t . y_{i}=c}\left[\mathbb{F}_{i}\left(f_{k j}\right)-m_{\mathrm{o}}\left(f_{k j}, c\right)\right]^{2}} .
$$

The mean and standard deviation of hypothesis $H_{1}$ can be computed as

$$
\begin{aligned}
m_{1}\left(f_{k j}, c\right) & =\frac{1}{N_{1}} \sum_{\forall i, s . t . y_{i} \neq c} \mathbb{F}_{i}\left(f_{k j}\right), \\
\sigma_{1}\left(f_{k j}, c\right) & =\sqrt{\frac{1}{N_{1}} \sum_{\forall i, s . t . y_{i} \neq c}\left[\mathbb{F}_{i}\left(f_{k j}\right)-m_{1}\left(f_{k j}, c\right)\right]^{2}} .
\end{aligned}
$$

To decide whether a filter is activated, we should choose a threshold denoted by $t$. Then, the false positive is the probability of the Gaussian distribution $\mathbb{N}\left(m_{1}, \sigma_{1}\right)$ falls in $[t,+\infty)$. The false negative is the probability of the Gaussian distribution $\mathbb{N}\left(m_{0}, \sigma_{0}\right)$ fall in $(-\infty, t]$. The sum of these two error types can be written as:

$$
\begin{aligned}
\mathbb{Q}_{k j}(t, c)= & \int_{-\infty}^{t} \mathbb{N}\left(m_{\circ}\left(f_{k j}, c\right), \sigma_{\circ}\left(f_{k j}, c\right)\right) \\
& +\int_{t}^{\infty} \mathbb{N}\left(m_{1}\left(f_{k j}, c\right), \sigma_{1}\left(f_{k j}, c\right)\right),
\end{aligned}
$$

It is typical to set

$$
t=\frac{1}{2}\left[m_{\mathrm{o}}\left(f_{k j}, c\right)+m_{1}\left(f_{k j}, c\right)\right] .
$$

The specific object class that minimizes $\mathbb{Q}_{k j}(t, c)$ in equation (5) is a candidate class for us to search for its GMC response. Mathematically, we have

$$
c_{g m c}=\underset{c \in \mathbb{C}}{\arg \min } \mathbb{Q}(t, c) .
$$

Finally, we define the GCM of filter $f_{k j}$ with respect to its corresponding $c_{g m c}$ as:

$$
\mathbb{G} \mathbb{C M}\left(f_{k j}\right)=\frac{\mathbb{Q}_{k j}\left(t, c_{g m c}\right)}{\mathbb{E}_{j}\left\{\mathbb{Q}_{k j}\left(t, c_{g m c}\right)\right\}} / \frac{m_{\circ}\left(f_{k j}, c_{g m c}\right)}{\mathbb{E}_{j}\left\{m_{\circ}\left(f_{k j}, c_{g m c}\right)\right\}} .
$$

The numerator and the denominator of GCM in equation (8) are the normalized decision error and the normalized mean of the null hypothesis, respectively. Intuitively, a GMC-like feature should have a higher mean response, $m_{0}\left(f_{k j}, c\right)$, due to the higher correlation between objects of the same class. Besides, it should have a lower decision error $\mathbb{Q}_{k j}(t, c)$ since it can differentiate positive and negative samples at higher accuracy. As a result, GCM approaches o and a larger number for GMC-like and non-GMC-like features, respectively. This is supported by experimental results given in Fig. 2, where we show both the mean response value and the decision error of 256 conv $_{5}$ features in a fast-RCNN trained CaffeNet with 40,000 iterations. Only three filters match the above description and the GCM successfully picks them out.

The GCM provides an effective feature evaluation tool based on the statistics of the response values of a filter given different inputs. Its validity is verified via applying Gaussian tests to the response values extracted from Pascal [38] dataset. It is also more thorough than evaluating the filter by APs since the GCM can model the underlining statistics of the filter (rather than testing the filter with a dataset). However, GCM is limited to the examination of a single filter. Thus, a new metric is needed for evaluating the group discriminative ability of a number of filters.

\section{B) Cluster purity measurement}

The CPM is proposed to evaluate how multiple filters perform jointly in differentiating object classes. As compared with the AP evaluation method introduced in [4], the CPM metric does not require the separate training of an SVM.

To evaluate the CPM score on a set of $n$ filters $f_{\vec{k}, \vec{j} \in \mathbb{J}}$ in the $k$ th convolution layer (』 represents the set of filter indexes), the testing samples $\left(\vec{x}_{i}, y_{i}\right)$ are organized into two groups, $\Omega_{c}$ and $\Omega_{\psi}$. The first group contains testing samples belonging to class $c$ while the second group contains testing samples not belonging to class $c$. In testing, the filter responses vector, $\overrightarrow{\mathbb{F}}_{i}\left(f_{\overrightarrow{k j}}\right)$, corresponding to the selected set of filters can be obtained at the $\operatorname{conv}_{k}$ layer. The responses vectors are

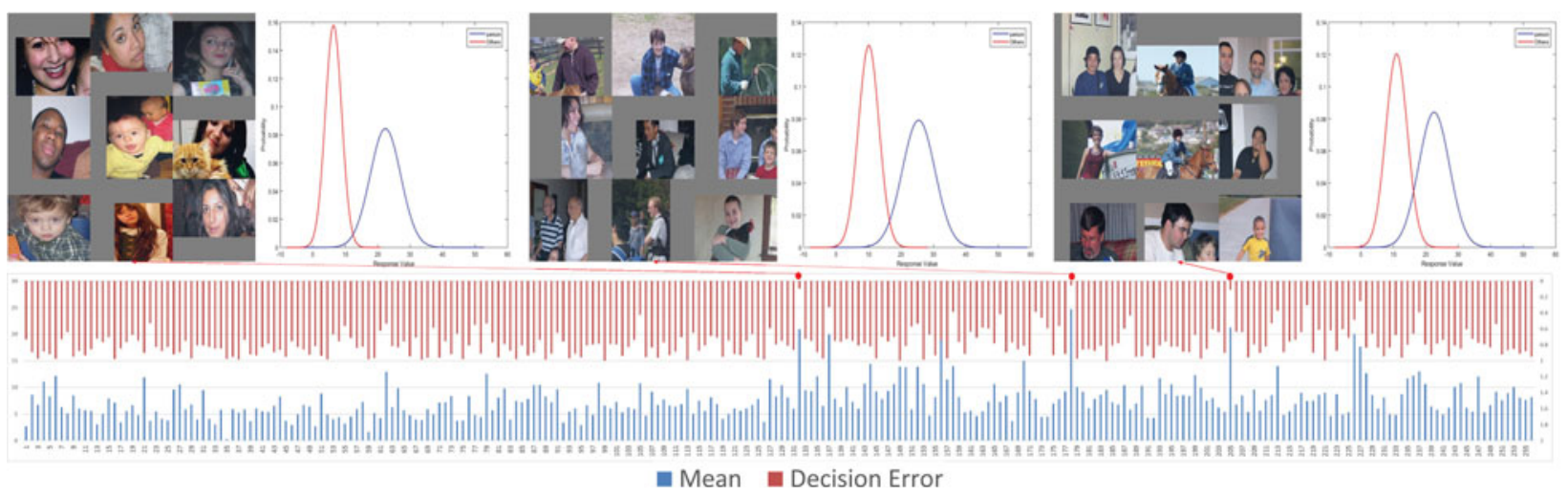

Fig. 2. The red and blue bars correspond to the decision error, $\mathbb{Q}_{k j}(t, c)$, and the null mean response, $m_{0}\left(f_{k j}, c\right)$, of the 256 conv ${ }_{5}$ filters of the fast-RCNN CaffeNet with 40,000 iterations, respectively. The blue bar is plotted bottom-up using the main vertical axis while the red bar is plotted top-down using the secondary vertical axis. The red dots indicate filters of indices 132, 178, and 205 (from left to right), which have the GCM score of 0.05 , 0.9 , and 0.17 , respectively. These filter responses have low decision errors so that they are selected as GMC-like features. The top nine activations and the Gaussian confusion plot for each of these three cases are presented for validation. 

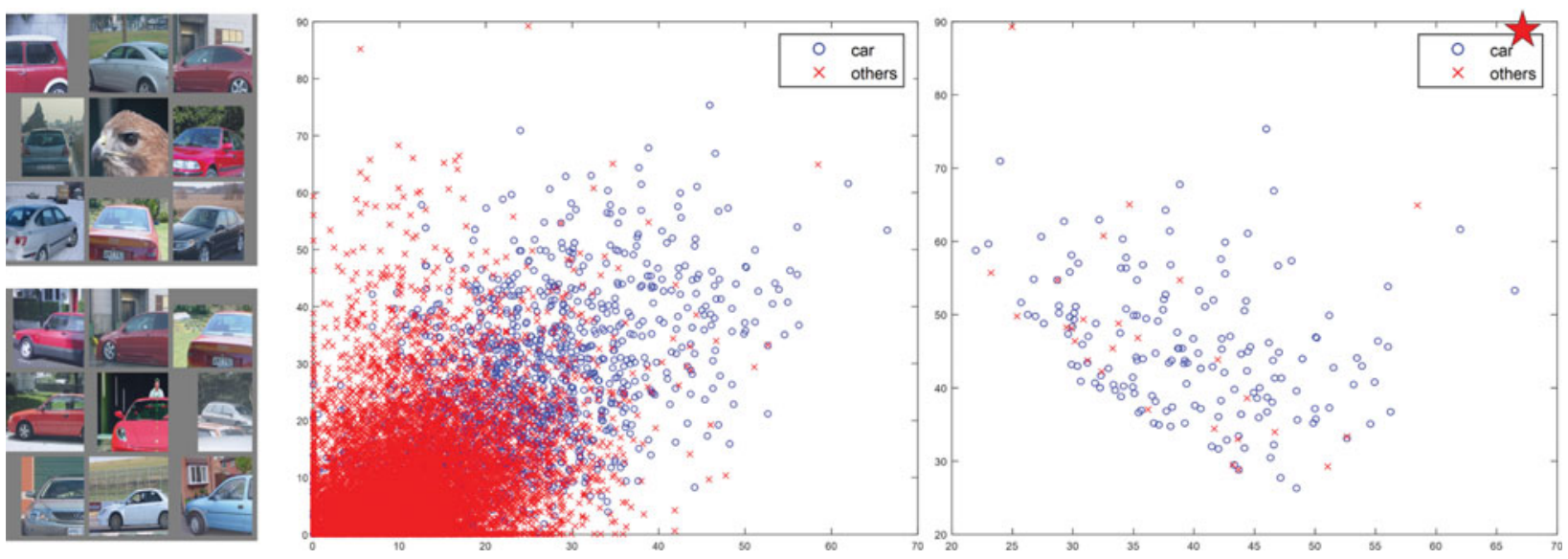

Fig. 3. The response vectors of filter 188 (top left) and 212 (bottom left) in the 4000 iteration CaffeNet model are plotted in the $2 \mathrm{D}$ space. The blue dots in the plot corresponds to response vectors obtained from testing samples of cars and the red dots corresponds response vectors obtained from others. The plot in the right takes the top 200 responses vectors that are closest to the point $P$ (shown as the star on the top right). The CPM score in this example is o.87.

$n$-dimensional vector. They can be split into two groups according to if the testing sample came from $\Omega_{c}$ or $\Omega_{\psi}$. An example of the response vector in the $2 \mathrm{D}$ case is shown in Fig. 3 .

In operation, the $\mathrm{CNN}$ prefers a high response from a filter when it 'fires' on an input. The recent success in the Binary network [39] further proves the fact that most of CNN filter's behavior can be considered as an ON/OFF switch. This motivates the CPM metric for a set of filters that better discriminates a certain object class jointly. For this reason, we define a characteristic point at $P=$ $\max _{i}\left\{\overrightarrow{\mathbb{F}}_{i}\left(f_{k j}\right)\right\}$ for an object class, where the max operation is taken over each dimension in the filter responses vector in the $n$-dimensional space.

As shown in Fig. 3, most points closer to $P$ correspond to the 'car' testing samples. To quantitatively measure this property, we define the CPM metric as a measure of the percentage of points corresponding to the target object class within the top $K$ closest points to point $P$. Mathematically, we have

$$
\mathbb{C P M}\left(\overrightarrow{\mathbb{F}}\left(f_{k j}\right), c\right)=\frac{N_{c}}{K}
$$

where $N_{c}$ is the number of responses vectors $\overrightarrow{\mathbb{F}}_{i}\left(f_{\vec{k}}\right)$, which are within the top $K$ points closest to $P$, and correspond to testing samples with class label $y_{i}=c$. We set $K=200$ empirically in the experiment.

In practice, we use the GCM metric to rank features' discriminative ability on each object class individually and, then, use the CPM metric to evaluate the joint discriminative ability of a selected group of features that perform well on a certain object class.
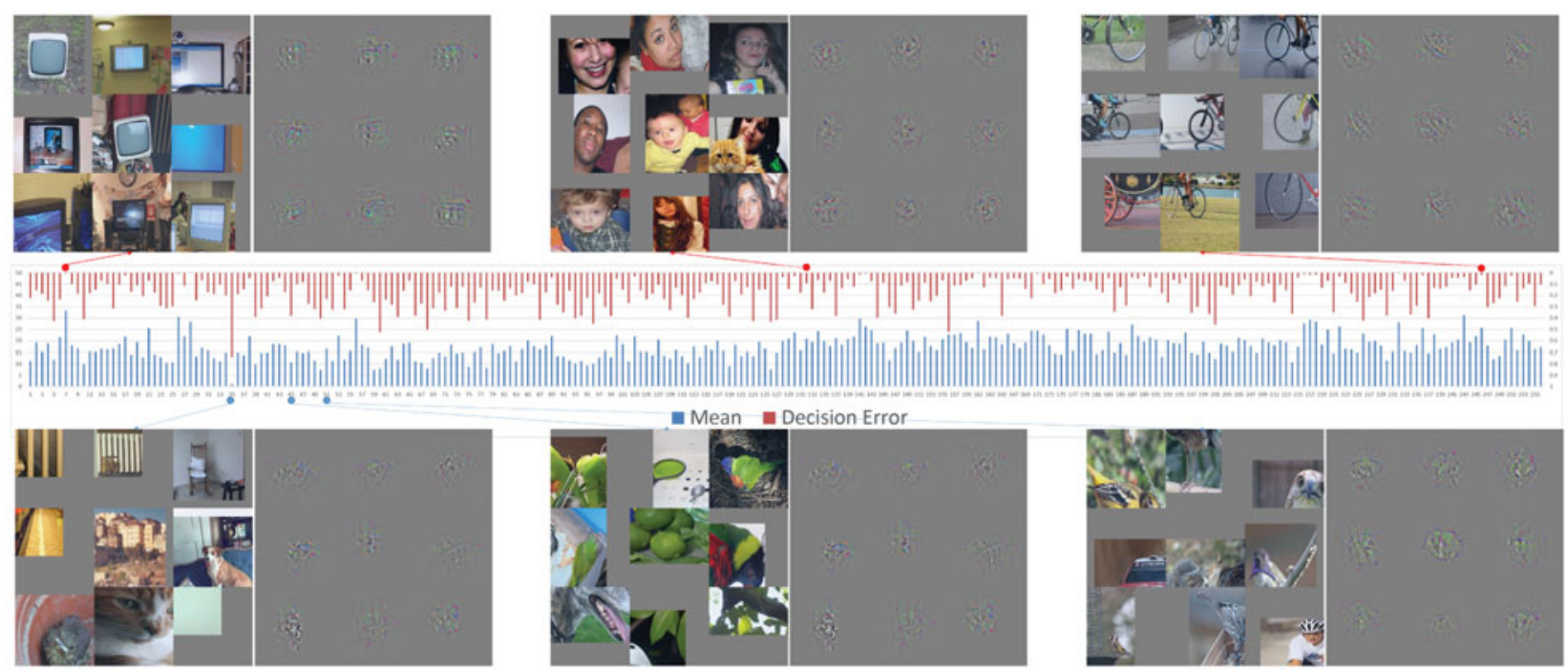

Fig. 4. The red and blue bars correspond to the decision error, $\min _{c} \mathbb{Q}_{k j}(t, c)$, and the null mean response value, $m_{0}\left(f_{k j}, c\right)$, of the 256 conv $v_{5}$ filters of the fast-RCNN CaffeNet with 40,00o iterations, respectively. (Note that these bars are different from those in Fig. 2, where $\mathbb{Q}_{k j}(t, c)$ and $m_{\mathrm{o}}\left(f_{k j}, c\right)$ were plotted for $c=$ "person"). The red bar is plotted top-down using the secondary vertical axis while the blue bar is plotted bottom-up using the main vertical axis. The red dots highlight filters 7, 132, and 246 from left to right. These filters have low decision errors and are selected as GMC-like features. The blue dots highlight filters 35 , 45, and 51. These filters have higher decision errors and their responses are selected as none-GMC-like features. The top nine activations and their deconv results are presented to validate whether it is a GMC-like or none-GMC-like feature. 

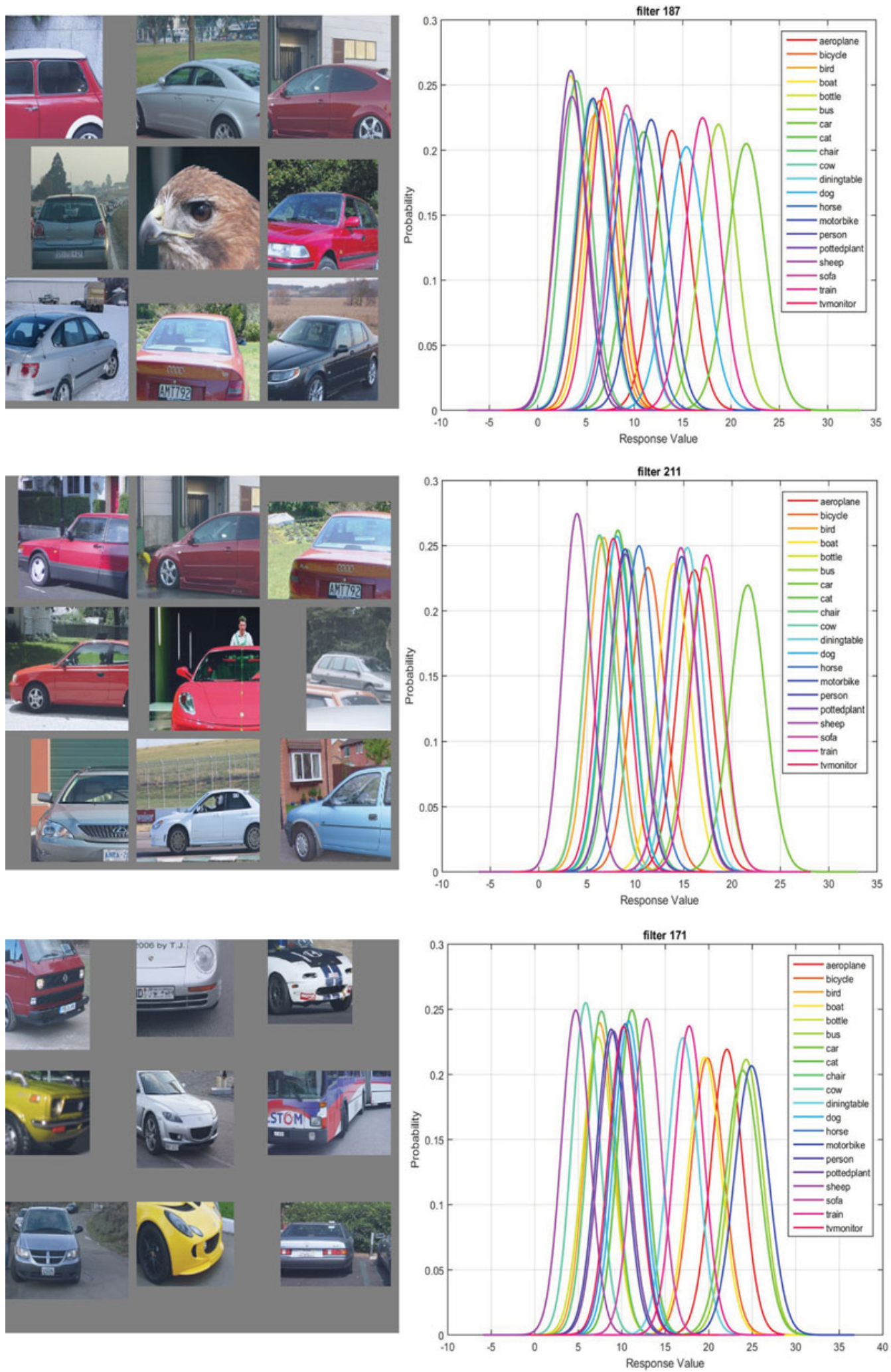

Fig. 5. From left to right: the top nine activations and the 20 classes Gaussian confusion plot of filters 188, 212, and 172 in conv 5 of the fast-RCNN CaffeNet with 40,0oo iterations. The CPM score is 0.54 for filter 188 alone, o. 86 for filter 188 and filter 212 - it reaches o.9o for the three filters group. The filters correspond to the tail, window, and head of the car, respectively. 

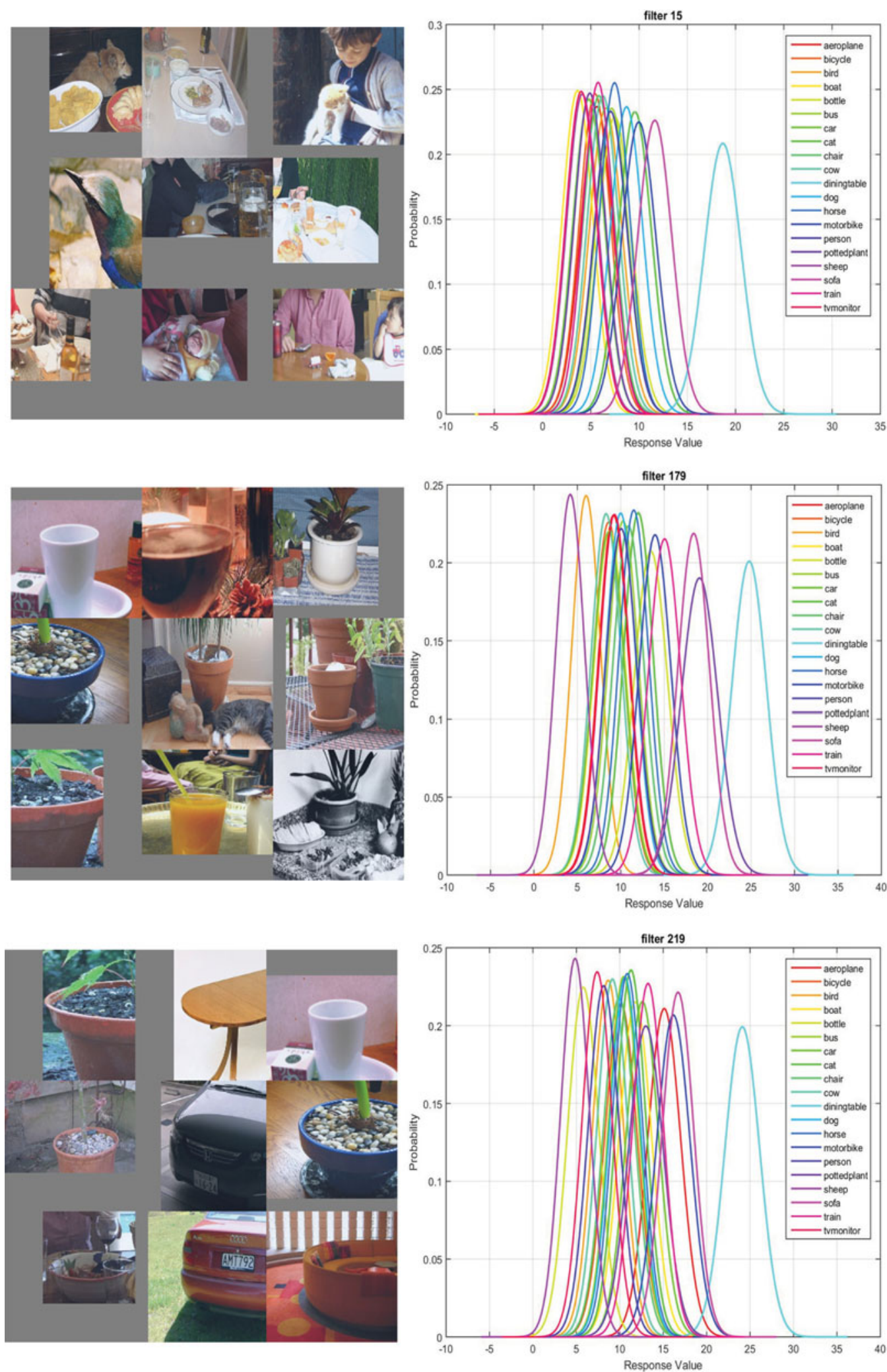

Fig. 6. From left to right: the top nine activations and the 20 classes Gaussian confusion plot of filters 16,180 , and 220 in conv 5 of the fast-RCNN CaffeNet Model with 40,00o iterations. The CPM score is o.36. These filters correspond to dishes, cups, an ellipse contour, respectively. Filters 16 and 180 are not related to a dining table itself but rather objects placed on top of it. 

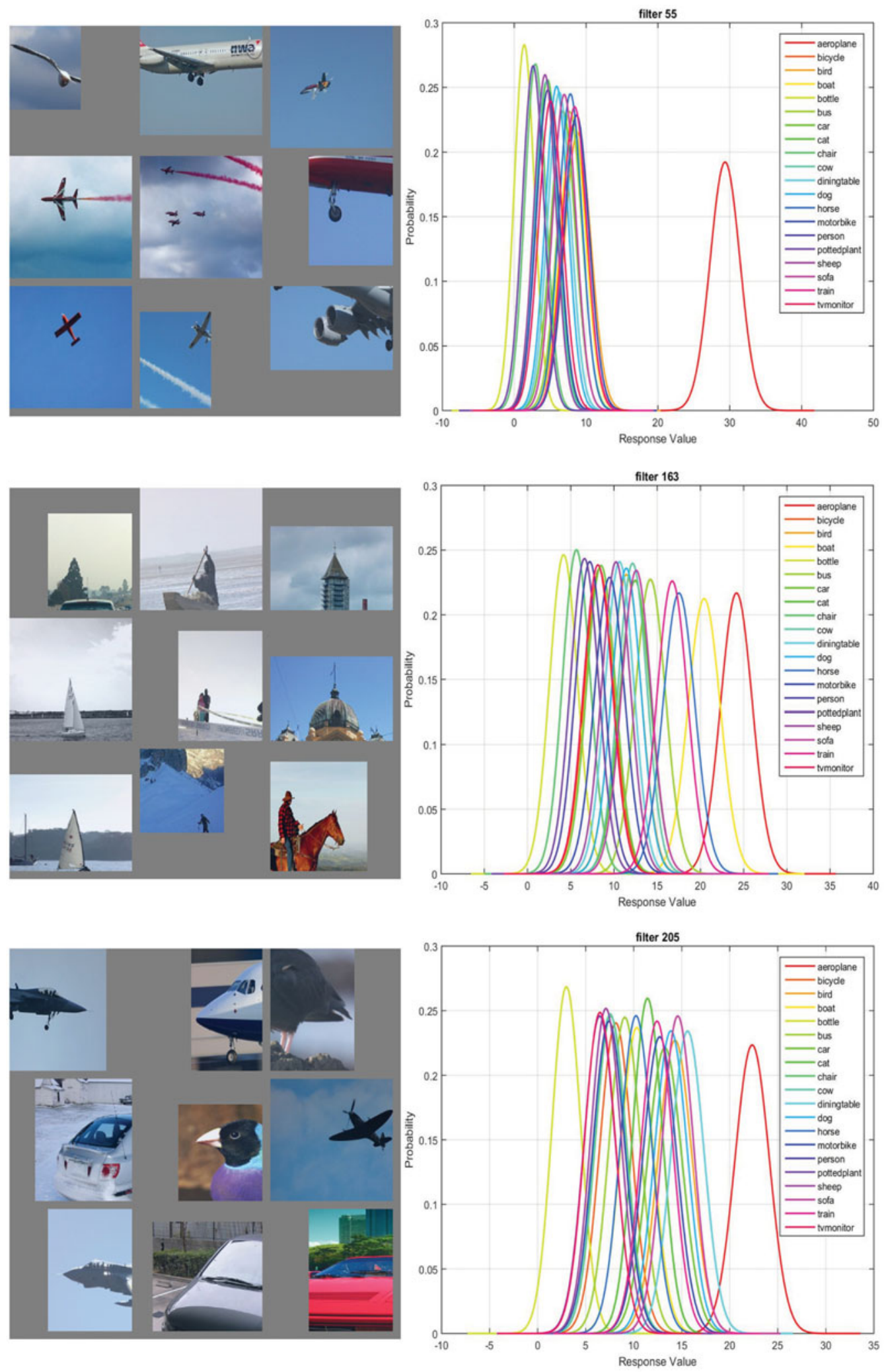

Fig. 7. The top nine activations and the 20 classes Gaussian confusion plot of filters 56, 164, and 206 in conv 5 of the fast-RCNN CaffeNet Model with 40,000 iterations are shown from left to right. The CPM score is 0.61 . These filters are all dedicated to detecting blue or gray color in the input, which are the typical background associated with "airplanes". 


\section{EXPERIMENTAL RESULTS}

We use the latest fast-RCNN $[9,40]$ implementation as the baseline. Since our objective is to analyze trained features, we disable the bounding box regression to avoid confusion. The analyzed network structure is the CaffeNet and VGG_M_1024, pretrained with the ImageNet CLS dataset [41]. To visualize filter responses (i.e., features) and top activations, we use the deconv network [3] and visualization toolbox from [11]. Furthermore, deconv images and top nine activations are extracted separately using the PASCALVOC 2012 dataset with the whole image as the ROI. To save space, we also generate the 20 classes Gaussian confusion plot by combining all 20 individual Gaussian confusion plots.

\section{A) Evaluating GMC features}

Our experiment begins with applying the two developed metrics, GCM and CPM, to the conv $_{5}$ features extracted from the CaffeNet. The fast-RCNN does not crop out ROIs until it reaches the fully connected layer. Thus, we created a new testing configuration, where the network ends at the ROI-pooling layer to obtain network responses at a given ROI. The ROI-pooling layer will output one maximum response value for each filter, which is used to evaluate whether the filter is activated or not. That is, for all 256 filters in the $\mathrm{conv}_{5}$ layer, we have a $256-\mathrm{D}$ feature vector to denote the response of each of the filters.

A few examples of GMC and none-GMC like features are demonstrated in Fig. 4, where the top nine activations of the GMC-like features are all of the same object classes, and their GCM values indicate that these filters do not confuse other object classes. The activations of none-GMC-like features vary on the other hand. From left to right, filter 35 seems to look for the vertical line structure, filter 45 seems to look for the green color, filter 51 seems to look for the defocused background. Due to the space limit in the paper, the full filter GCM results are included in the supplemental material as the appendix.

\section{B) Evaluating GMC feature groups}

Besides individual GMC-like features, the proposed CPM can find some interesting GMC-like feature groups. The top nine activations and the 20 classes Gaussian confusion plot of filter 188, 212, and 172 in conv $_{5}$ are shown in Fig. 5 .

The three filters are the top contributors of a feature group for the "car" object. Individually, each of them has a high decision error. They can get confused with different object classes (e.g., "bird" and "bus"). However, when the three work together, the discriminative power improves a lot and the feature group will mostly respond to the "car" images. As compared with an individual GMC, another advantage of the feature group is its robustness against occlusion. The GMC-like features are extremely sensitive to occlusion. This is a real issue in general object detection since objects are often partially occluded in images. Since the GMC-like feature group can respond to a subset of object's components, its detection performance will be more robust when an occlusion occurs.

\section{C) Evaluating context features}

Detection of a "dining table" and a "chair" is interesting as there is no clear definition of the object. Before applying the CNN to object detection, the APs of using the DPM [42] in detecting a dining table and a chair are both low. They are 14.7 and $17.2 \%$, respectively, in the PASCAL-VOC 2012 dataset [38]. Recently, the fast-RCNN significantly boosts the corresponding performance to 40.7 and $67.9 \%$. We observe from our experiment that the difference in the performance gain is due to "context features" trained by the network.

By identifying a group of features that corresponds to detecting "dining table" and plotting their top nine activations and the 20 classes Gaussian confusion plot in Fig. 6, we see that the key information used in detecting a dining table is cups and/or dishes placed on top of it. That is, while the DPM detector attempts to model the shape or contour of an object, there is little success in man-made objects due


Fig. 8. From left to right: (1) the top nine activations of filter 216 in conv 5 layer of the 40000 iteration CaffeNet model, and (2) the Gaussian confusion plot of the bicycle versus others, the motorbike versus others, and the car versus others. 

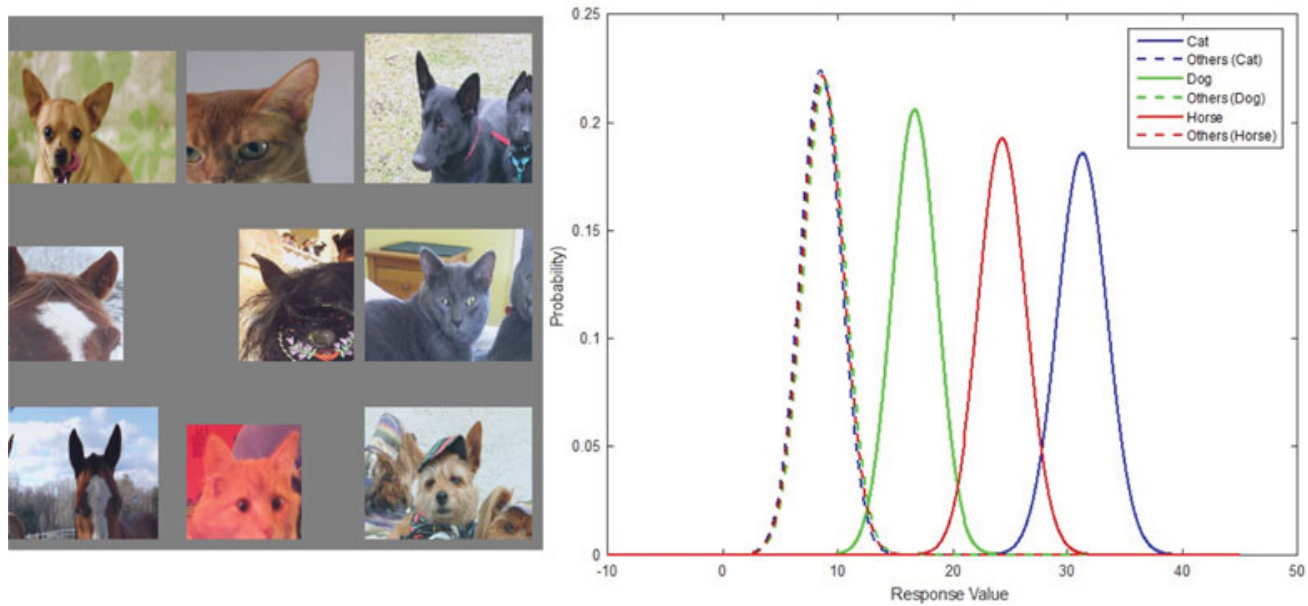

Fig. 9. From left to right: (1) the top nine activations of filter 141 in conv $_{5}$ layer of the 40000 iteration CaffeNet model, and (2) the Gaussian confusion plot of the cat versus others, the horse versus others, and the dog versus others.
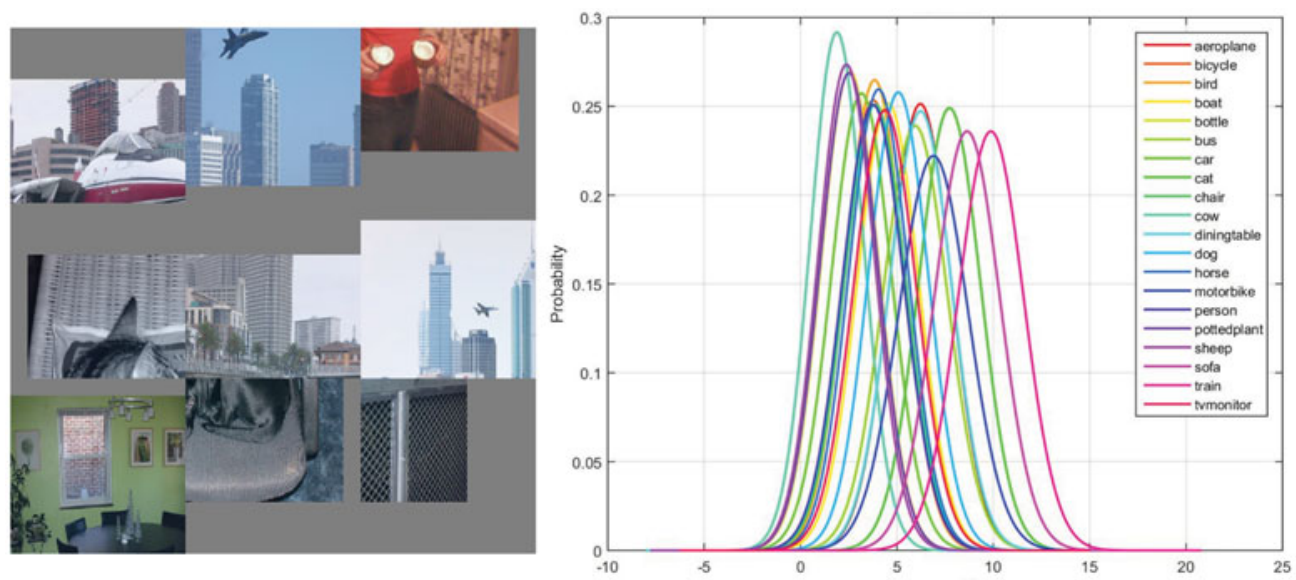

Fig. 10. From left to right: (1) the top nine activations of filter 214 in conv 5 layer of the 40000 iteration CaffeNet model, and (2) the Gaussian confusion plot of all 20 object classes.
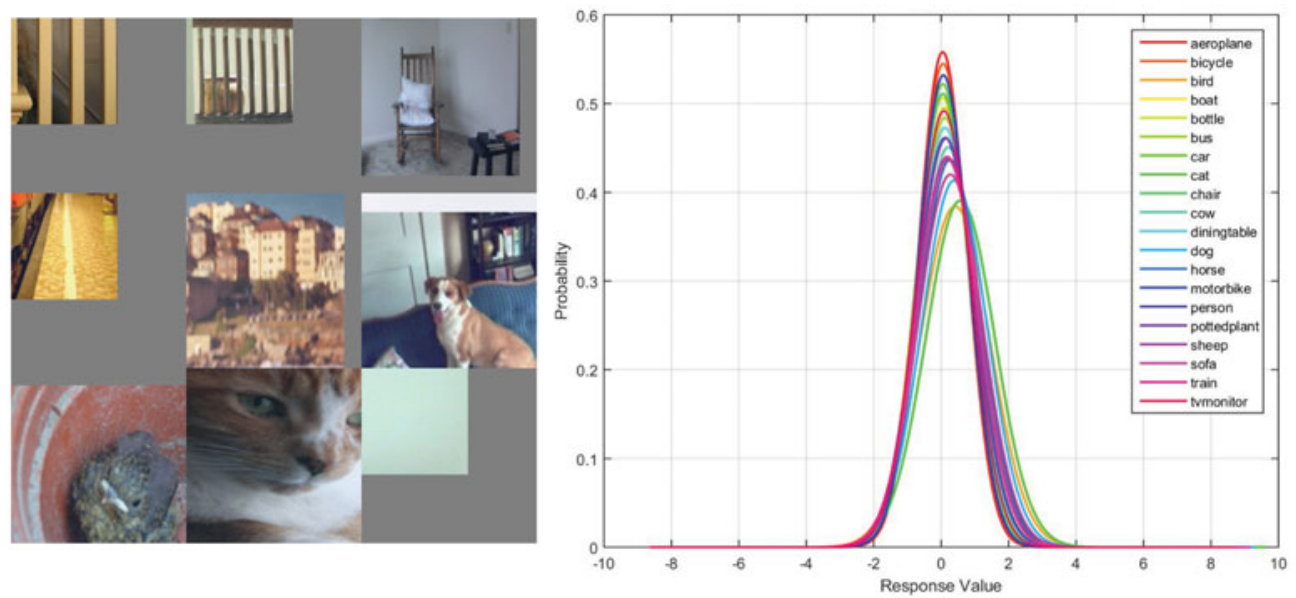

Fig. 11. From left to right: (1) the top nine activations of filter 35 in conv 5 layer of the 40000 iteration CaffeNet model, and (2) the Gaussian confusion plot of all 20 object classes.

to intra-class variation. The $\mathrm{CNN}$ approaches this problem by summarizing the function of the dining table, which is to hold cups or dishes on top of it. This is quite close to how humans define a dining table: "an object to put dishes on".
Besides context features that correspond to the function of an object, $\mathrm{CNN}$ also learns the context features that associate with the most probable background of an object. In Fig. 7, the three features have a CPM score of 0.61. However, the top nine activations and the 20 classes Gaussian 

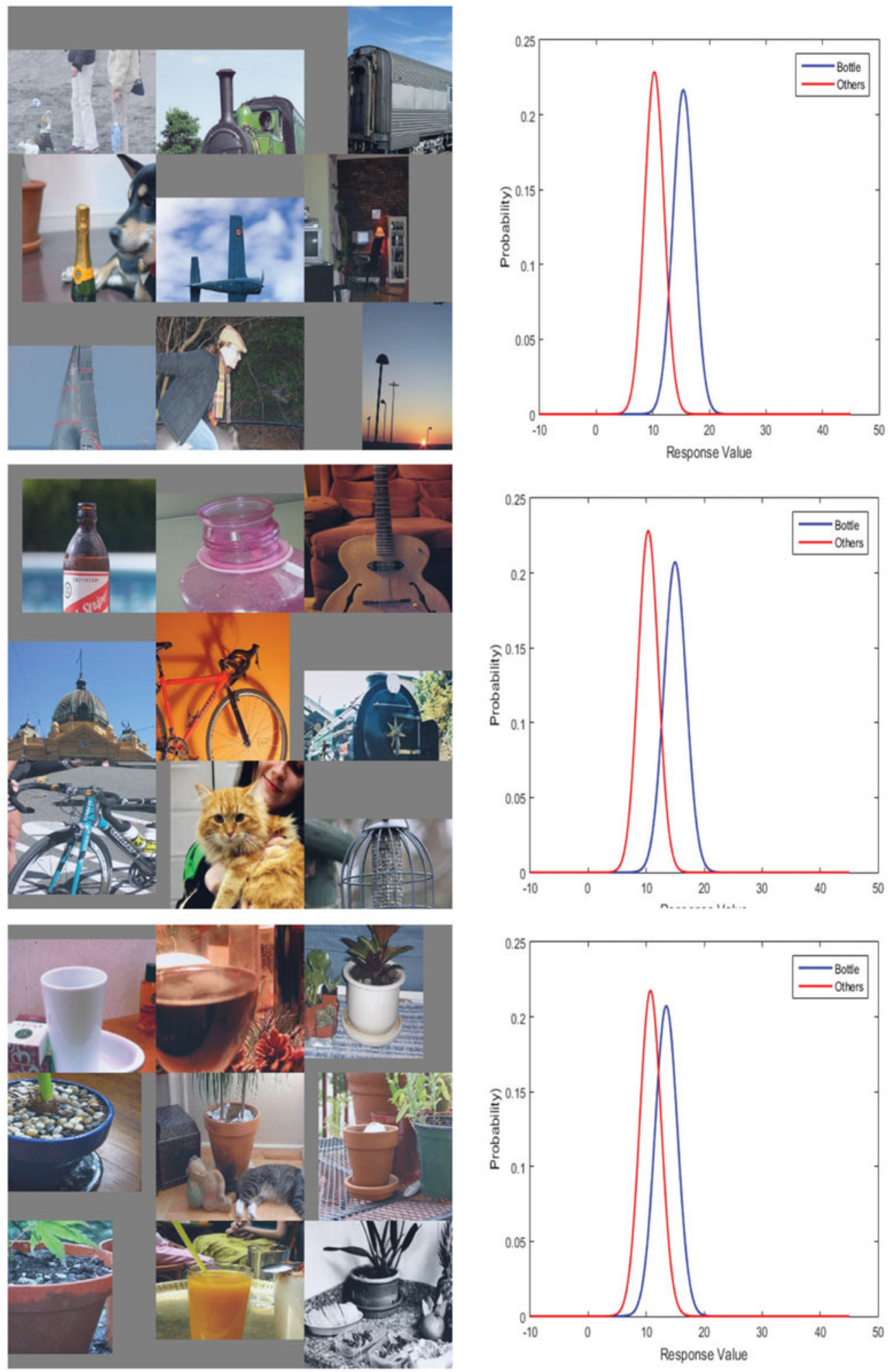

Fig. 12. The top three filters in the CaffeNet trained to detect the "bottle" object. Their corresponding Gaussian confusion plots are shown under the top nine activations.

confusion plot both confirm that the features are not learned to detect 'airplanes' but the background.

\section{D) Evaluating shared features}

The CNN also learns shared features that have the superior discriminative ability over a few object classes. In
Fig. 8, the top nine activations and the Gaussian confusion plot verify that the underlying feature tries to detect the wheel of a motorbike or a bicycle. Although the top nine activations include examples of the wheel of cars, the Gaussian confusion plot indicates that the filter is less capable of differentiating cars from others. As shown, the feature can differentiate motorbikes and bicycles from 
other objects, but cannot separate them well. This example demonstrates the first type of shared feature, which corresponds to certain shared characteristics across different object classes such as a common part of different objects.

There is another type of shared features, which not only corresponds to multiple object classes but has the
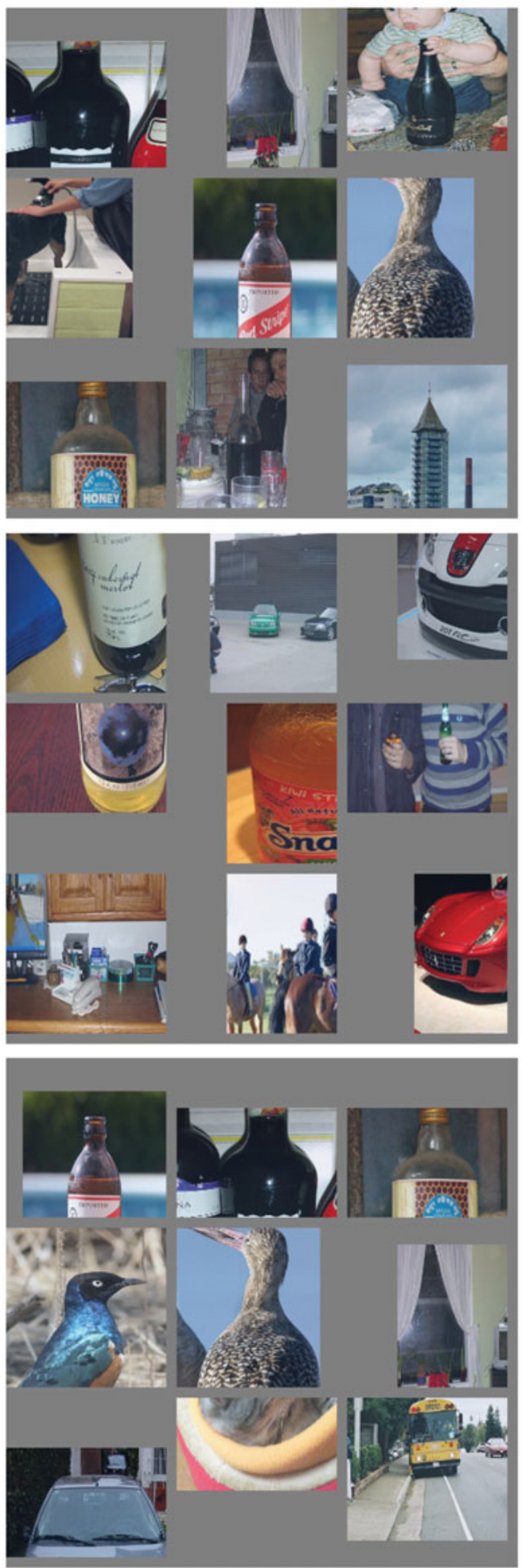

discriminative ability to separate object classes. In Fig. 9, the top nine activations and the Gaussian confusion plot verify that the feature tries to detect the ear of cats, dogs or horses. Unlike the previous example, this feature has different response value ranges for the three object classes and can separate them with small decision errors. Since this feature's discriminative ability is closely related to the actual response
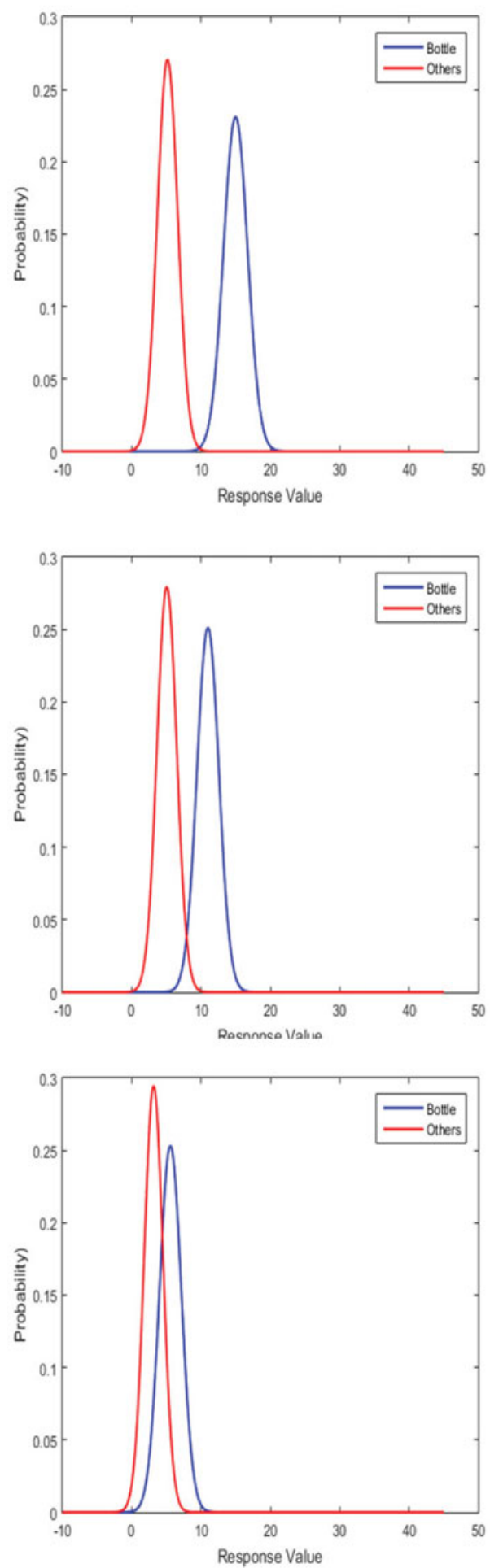

Fig. 13. The top three filters in the VGG_M_1024 (right) trained to detect the "bottle" object. Their corresponding Gaussian confusion plots are shown under the top nine activations. 
values, it will be deprecated in the binary network [39]. This explains why the binary network could only achieve similar performance as the floating point network but could not outperform it.

\section{E) Other features}

Besides the features that correspond to one or several object classes, the CNN learns features that seem to have little discriminative ability between classes. Usually, these features correspond to generic texture, shape or color. In Fig. 10, the top nine activations indicate that the filter is looking for mesh-like texture. The corresponding Gaussian confusion plot indicates that the feature can hardly differentiate between any classes by itself.

There is also another type of filter, which is confirmed to be an "unused" filter for classification. An example is shown in Fig. 11, where the top nine activations indicate that the filter is looking for parallel vertical patterns. However, the Gaussian confusion plot of all 20 object class shows that the feature has the same response range for all object classes. By examining the $\mathrm{FC}_{1}$ weight assigned to this $\operatorname{conv}_{5}$ feature, we see that it is almost ignored since the weight for this filter is negligible. Although these type of features seem to have little use in classification, they contribute strongly for generation tasks. The recent success in style transfer [43] relies heavily on these generic features as they contribute to the computation of the "style loss".

\section{F) Comparison between VGG_M_1024 and CaffeNet}

The VGG_M_1024 network structure used in the fastRCNN benchmark is selected for comparison with the CaffeNet. The two networks start from the same 96 conv $_{1}$ features, which are combined into 256 features in $\operatorname{conv}_{2}$. From that point on, while the CaffeNet has 384, 384, and 256 features in $\operatorname{conv}_{3}, \operatorname{conv}_{4}$, and conv 5 layers, respectively, the VGG_M_1024 has 512, 512, and 512 features in its corresponding layers. The CaffeNet uses grouping to divide filters into two groups due to the GPU memory limit at their development time, which results in divided feature representation that may be less optimal as compared with VGG_M_1024 where all features are combined.

Due to the larger capacity in deeper convolution layers, the VGG_M_1024 model has a higher chance to learn GMC-like features, and the feature representation learned is more comprehensive and discriminative. An example is the "bottle" object class, where the CaffeNet has no GMClike feature while the VGG_M_1024 have two GMC-like features. As shown in Figs 12 and 13, the most discriminative features trained by the CaffeNet are not GMC-like features. Thus, the decision error is large. In contrast, there are two GMC-like features trained by the VGG_M_1024, leading to low decision errors. In general, features trained from VGG_M_1024 tend to be more discriminative, and more GMC-like features can be found in VGG_M_1024. A

Table 1. The CPM scores of the top 5 features in the CaffeNet and the VGG_M_1024 for each object class.

\begin{tabular}{|c|c|c|c|c|c|c|c|c|c|c|c|c|c|c|c|c|c|c|c|c|}
\hline Network & eroplan & Bicycle & Bird & Boat & Bottle & Bus & Car & Cat & Chair & Cow & Table & Dog & Horse & Motorbil & Person & Plant & Sheep & Sofa & Train & $\mathrm{Tv}$ \\
\hline CaffeNet & 0.67 & 0.54 & 0.27 & 0.51 & 0.21 & 0.3 & 0.92 & 0.70 & 0.23 & 0.31 & 0.33 & 0.66 & 0.61 & 0.46 & 0.89 & 0.45 & 0.51 & 0.36 & 0.57 & 0.63 \\
\hline VGG & 0.70 & 0.60 & 0.59 & 0.61 & 0.37 & 0.49 & 0.95 & 0.86 & 0.50 & 0.47 & 0.55 & 0.77 & 0.42 & 0.49 & 0.91 & 0.67 & 0.53 & 0.46 & 0.47 & 0.68 \\
\hline
\end{tabular}

Table 2. The functionality summary of $\operatorname{conv}_{5}$ filters trained by the CaffeNet and the VGG_M_1024.




summary of the feature representation for each object class is concluded in Table 1, where the CPM scores of the top 5 features for each object class are compared between the CaffeNet and the VGG_M_1024. The comparison further verifies that deeper networks learn better feature representation.

\section{G) Complete filter profile}

After analyzing the complete set of $\mathrm{CNN}$ trained features, we summarize different types of features trained by the CaffeNet and the VGG_M_1024 in Table 2, where the "1 class GMC" features correspond to features that differentiate one object class from the others (e.g., the dog example in Fig. 1), the "shared and distinctive" features correspond to features that differentiate several object classes from the others but cannot differentiate within these classes (e.g., example in Fig. 9), the "shared and not distinctive" feature correspond to features that differentiate several object classes from the others and differentiate within these classes (e.g., example in Fig. 8), and the 'other' features include the rest of the more generic features.

\section{H) Summary}

We have identified the following feature types learned by CNNs:

(i) GMC-like features or feature groups;

(ii) context features related to the function or most probable background of the object;

(iii) distinctive or non-distinctive shared features;

(iv) non-discriminant features.

We also verified that deeper networks can learn more GMClike features so as to yield better performance.

\section{CONCLUSION}

Two effective metrics (i.e., GCM and CPM) to evaluate trained features of CNNs were proposed in this work. Thorough studies were conducted on deep features learned from the CaffeNet and VGG_M_1024 network based on these metrics. We identified different feature types, compared their discriminative ability, and analyzed the advantages of the deeper network structure in the experiment. Finally, we provided a full conv $v_{5}$ feature profile for both the CaffeNet and VGG_M_1024 to conclude our study.

Understanding $\mathrm{CNN}$ features can help understand what exactly has been learned in the network. Our study could potentially contribute to the development of automatic algorithms to determine the optimal network structure. It provides a quantitative evaluation of learned networks by introducing a loss term that is related to the CNN structure [44].

\section{ACKNOWLEDGEMENT}

Computation for the work described in this paper was supported by the University of Southern Californiass Center for High-Performance Computing (hpc.usc.edu).

\section{REFERENCES}

[1] Mahendran, A.; Vedaldi, A.: Understanding deep image representations by inverting them, in IEEE Conf. on Computer Vision and Pattern Recognition, 2015.

[2] Wei, D.; Zhou, B.; Torralba, A.; Freeman, W.: Understanding IntraClass Knowledge Inside CNN, ArXiv preprint ArXiv: 1507.02379, (2015).

[3] Zeiler, M.D.; Fergus, R.: Visualizing and understanding convolutional networks, in European Conf. on Computer Vision, 2014.

[4] Agrawal, P.; Girshick, R.; Malik, J.: Analyzing the performance of multilayer neural networks for object recognition, in European Conf. on Computer Vision, 2014

[5] Quiroga, R.Q.; Reddy, L.; Kreiman, G.; Koch, C.; Fried, I.: Invariant visual representation by single neurons in the human brain. Nature 435 (7045) (2005), 1102.

[6] Le Q. et al:: Building high-level features using large scale unsupervised learning, in Int. Conf. in Machine Learning, 2012.

[7] Hu, J.; Deng, W.; Guo, J.: Online regression of grandmother-cell responses with visual experience learning for face recognition, in Int. Conf. on Pattern Recognition, 2014.

[8] Wersing, H.; Korner, E.: Learning optimized features for hierarchical models of invariant object recognition. Neural. Comput., 15 (7) (2003), 1559-1588.

[9] Girshick, R.: Fast R-CNN, arXiv preprint arXiv:1504.08083, 2015.

[10] Simonyan, K.; Vedaldi, A.; Zisserman, A.: Learning local feature descriptors using convex optimisation. IEEE Trans. Pattern Anal. Mach. Intell., 36 (8) (2014), 1573-1585.

[11] Yosinski, J.; Clune, J.; Nguyen, A.; Fuchs, T.; Lipson, H.: Understanding neural networks through deep visualization, in Deep Learning Workshop of Int. Conf. on Machine Learning, 2015.

[12] Girshick, R.; Donahue, J.; Darrell, T.; Malik, J.: Rich feature hierarchies for accurate object detection and semantic segmentation, in IEEE Conf. on Computer Vision and Pattern Recognition, 2014.

[13] Krizhevsky, A.; Sutskever, I.; Hinton, G.E.: Imagenet classification with deep convolutional neural networks, in Neural Information Processing Systems, 2012.

[14] Li, Y.; Liu, L.; Shen, C.; van den Hengel, A., : Mid-level deep pattern mining, in IEEE Conf. on Computer Vision and Pattern Recognition, 2015.

[15] Bengio, Y.; Goodfellow, I.J.; Courville, A.: Deep Learning, 2015, http://www.iro.umontreal.ca/ bengioy/dlbook, book in preparation for MIT Press

[16] Srivastava, R.K.; Masci, J.; Gomez, F.; Schmidhuber, J.: UNDERSTANDING LOCALLY COMPETITIVE NETWORKS, ArXiv preprint ArXiv: 1410.1165, 2015.

[17] Girshick, R.; Iandola, F.; Darrell, T.; Malik, J.: Deformable part models are convolutional neural networks, in IEEE Conf. on Computer Vision and Pattern Recognition, 2015.

[18] Zhou, B.; Khosla, A.; Lapedriza, A.; Oliva, A.; Torralba, A.: Object detectors emerge in deep scene CNNs, in Int. Conf. on Learning Representations, 2015. 
[19] Kuo, C.C.J.: Understanding convolutional neural networks with a mathematical model. J. Vis. Commun. Image. Represent., $4 \mathbf{1}$ (2016), 406-413, http://www.sciencedirect.com/science/article/pii/ S1047320316302267

[20] Kuo, C.C.J.: The CNN as a guided multilayer RECOS Transform [Lecture Notes]. IEEE Signal Process. Mag., 34 (3) (2017), 81-89.

[21] Szegedy C. et al.: Intriguing properties of neural networks, in Int. Conf. on Learning Representations, 2014.

[22] Goodfellow, I.J.; Shlens, J.; Szegedy, C.: Explaining and harnessing adversarial examples, in Int. Conf. on Learning Representations, 2015.

[23] Xie, C.; Wang, J.; Zhang, Z.; Zhou, Y.; Xie, L.; Yuille, A.: Adversarial examples for semantic segmentation and object detection, in 2017 IEEE Int. Conf. on Computer Vision (ICCV), October 2017, 1378-1387.

[24] Zhang, Q.; Wang, W.; Zhu, S.C.: Examining cnn representations with respect to dataset bias, in AAAI Conf. on Artificial Intelligence (AAAI), 2018.

[25] Zhang, Q.; Cao, R.; Shi, F.; Wu, Y.N.; Zhu, S.C.: Interpreting CNN knowledge via an explanatory graph, in AAAI Conf. on Artificial Intelligence (AAAI), 2018.

[26] Zhang, Q.; Yang, Y.; Wu, Y.N.; Zhu, S.C.: Interpreting CNNs via decision trees, arXiv preprint arXiv:1802.00121, 2018.

[27] Sabour, S.; Frosst, N.; Hinton, G.E.: Dynamic routing between capsules, in Advances in Neural Information Processing Systems, 2017, 3856-3866.

[28] Hinton, G.E.; Krizhevsky, A.; Wang, S.D.: Transforming autoencoders, in Int. Conf. on Artificial Neural Networks, Springer, 2011, 44-51.

[29] LeCun, Y.; Cortes, C.; Burges, C.J.: The MNIST Database of handwritten digits, 2018, http://yann.lecun.com/exdb/mnist/.

[30] Chen, X.; Duan, Y.; Houthooft, R.; Schulman, J.; Sutskever, I.; Abbeel, P.: Infogan: Interpretable representation learning by information maximizing generative adversarial nets, in Advances in neural information processing systems, 2016, 2172-2180.

[31] Netzer, Y.; Wang, T.; Coates, A.; Bissacco, A.; Wu, B.; Ng, A.Y: Reading digits in natural images with unsupervised feature learning, in NIPS Workshop on Deep Learning and Unsupervised Feature Learning, 2011.

[32] Paysan, P.; Knothe, R.; Amberg, B.; Romdhani, S.; Vetter, T.: A 3D face model for pose and illumination invariant face recognition, in Advanced video and signal based surveillance, 2009. AVSS'09. Sixth IEEE Int. Conf. on, Ieee, 2009, 296-301.

[33] Aubry, M.; Maturana, D.; Efros, A.A.; Russell, B.C.; Sivic, J.: Seeing 3d chairs: exemplar part-based $2 \mathrm{~d}-3 \mathrm{~d}$ alignment using a large dataset of cad models, in Proceedings of the IEEE conference on computer vision and pattern recognition, 2014, 3762-3769.

[34] He, K.; Zhang, X.; Ren, S.; Sun, J.: Spatial Pyramid pooling in deep convolutional networks for visual recognition, in European Conf. on Computer Vision, 2014.

[35] Ren, S.; He, K.; Girshick, R.; Sun, J.: Faster R-CNN: towards real-time object detection with region proposal networks, in Neural Information Processing Systems, 2015.

[36] Simonyan, K.; Zisserman, A.: Very deep convolutional networks for large-scale image recognition, in Int. Conf. on Learning Representations, 2014.

[37] Szegedy C. et al.: Going deeper with convolutions, in IEEE Converence on Computer Vision and Pattern Recognition, 2015.

[38] Everingham, M.; Van Gool, L.; Williams, C.K.I.; Winn, J.; Zisserman, A.: The Pascal Visual Object Classes (VOC) challenge. Int. J. Comput. Vis., 88 (2) (2010), 303-338.
[39] Courbariaux, M.; Bengio, Y.; David, J.P.: BinaryConnect: training deep neural networks with binary weights during propagations, in Neural Information Processing Systems, 2015.

[40] Jia Y. et al.: Caffe: convolutional architecture for fast feature embedding, arXiv preprint arXiv:1408.5093, (2014)

[41] Russakovsky O. et al.: ImageNet large scale visual recognition challenge, in Int. Journal of Computer Vision, 2015.

[42] Felzenszwalb, P.F.; Girshick, R.B.; McAllester, D.; Ramanan, D.: Object detection with discriminatively trained part based models. IEEE Trans. Pattern Anal. Mach. Intell., 32 (9) (2010), 16271645 .

[43] Gatys, L.A.; Ecker, A.S.; Bethge, M.: A Neural Algorithm of Artistic Style, arXiv preprint arXiv:1508.06576, (2015).

[44] Liu C. et al.: Progressive neural architecture search, arXiv preprint arXiv:1712.00559, 2017.

Hao Xu received the B.S. in Electrical Engineering from Purdue University in 2010 and received his Ph.D. degree from University of Southern California in 2017. He joined Google after completing his Ph.D. studies. His research interest lies in $3 \mathrm{D}$ image/video rendering, $2 \mathrm{D} / 3 \mathrm{D}$ video conversion, and Object Detection.

Yueru Chen is a Ph.D. student at University of Southern California. She received her Bachelor's degree in Physics from the University of Science and Technology of China, China in June 2014. Her research interests include $3 \mathrm{D}$ retrieval and computer vision.

Ruiyuan Lin is a Ph.D. student at University of Southern California. She received her Bachelor's degree in Information Engineering from City University of Hong Kong in July 2016. Her research interests include computer vision and machine learning.

C.-C. Jay Kuo received his Ph.D. degree from the Massachusetts Institute of Technology in $1987 . \mathrm{He}$ is now with the University of Southern California (USC) as Director of the Media Communications Laboratory and Dean's Professor in Electrical Engineering-Systems. His research interests are in the areas of digital media processing, compression, communication and networking technologies. Dr. Kuo is a Fellow of AAAS, IEEE and SPIE. He has guided 140 students to their Ph.D. degrees and supervised 25 postdoctoral research fellows. Dr. Kuo is a co-author of about 260 journal papers, 900 conference papers, 14 books and 30 patents. Dr. Kuo received the 1992 National Science Foundation Young Investigator (NYI) Award, the 1993 National Science Foundation Presidential Faculty Fellow (PFF) Award, the 2010 Electronic Imaging Scientist of the Year Award, the 2010-11 Fulbright-Nokia Distinguished Chair in Information and Communications Technologies, the 2014 USC Northrop Grumman Excellence in Teaching Award, the 2016 USC Associates Award for Excellence in Teaching, the 2016 IEEE Computer Society Taylor L. Booth Education Award, the 2016 IEEE Circuits and Systems Society John Choma Education Award, the 2016 IS\&T Raymond C. Bowman Award, and the 2017 IEEE Leon K. Kirchmayer Graduate Teaching Award. 